\title{
Valores de glucosa-6-fosfato deshidrogenasa y su repercusión en el número de sospechas de tamiz neonatal
}

\section{Glucose-6-phosphate dehydrogenase values and their impact on the number of suspected neonatal screening}

\author{
Karla Maldonado-Silva, ${ }^{1}$ Mirna Angélica Hinojosa-Trejo, ${ }^{2}$ Isabel Ibarra-González, ${ }^{3}$ Marcela Vela-Amieva, ${ }^{4}$ Luz del \\ Alba Herrera-Pérez, ${ }^{5}$ Guillermo Caamal-Parra, ${ }^{5}$ Justo Eduardo Sulu-Huicab, ${ }^{5}$ Erika Paola García-Flores ${ }^{2}$
}

\begin{abstract}
Resumen
INTRODUCCIÓN: La deficiencia de glucosa-6-fosfato deshidrogenasa (dG6PD) es la enzimopatía hereditaria más frecuente en el mundo. La mayoría de los casos son asintomáticos y algunos Ilegan a padecer anemia hemolítica aguda, ictericia neonatal o anemia hemolítica crónica no esferocítica. En países de Asia y el Mediterráneo y con alta incidencia de malaria, se practica el tamiz neonatal para la dG6PD, pero no existe un consenso universal para su implementación.

OBJETIVO: Describir los valores de la actividad de G6PD en papel filtro en recién nacidos, analizar la prevalencia y la repercusión en el número de localizaciones a efectuar en el programa de tamiz neonatal nacional.

MATERIAL Y MÉTODOS: Estudio retrospectivo de los resultados del programa de tamiz neonatal de la Secretaría de Salud de México. Se analizó la actividad de la glucosa-6-fosfato deshidrogenasa dependiendo del tiempo de tránsito (tiempo trascurrido desde la obtención de la muestra de sangre del talón del recién nacido hasta su llegada al laboratorio). Se analizó la prevalencia según la actividad enzimática residual. RESULTADOS: De 1,076,918 recién nacidos tamizados, 343,272 tuvieron tiempo de tránsito adecuado ( $\leq 6$ días). La actividad enzimática desciende significativamente después de ese tiempo. La prevalencia nacional al nacimiento fue de $4.26 \%$, con porcentajes máximos en Veracruz, Nuevo León y Tabasco (21,20 y 15\%, respectiva-
\end{abstract} mente).

CONCLUSIÓN: $4.26 \%$ de los recién nacidos en México tienen dG6PD; es decir, un caso por cada 23 tamizados, lo que implica un elevado número de sujetos para localizar y evaluar en los programas de tamiz neonatal y en las instituciones que llevan a cabo el seguimiento clínico.

PALABRAS CLAVE: Glucosa 6-fosfato deshidrogenasa, deficiencia de glucosa 6-fosfato deshidrogenasa, tamiz neonatal, prevalencia.

Abstract

INTRODUCTION: Glucose-6-phosphate dehydrogenase (dG6PD) deficiency is the most common inherited blood enzymopathy worldwide. Most G6PD deficient subjects are asymptomatic, but some of them can develop acute hemolytic anemia, neonatal jaundice or non-spherocytic chronic hemolytic anemia. In some Asiatic and Mediterranean countries with a high incidence of malaria, the newborn screening (NBS) for dG6PD is performed, but there is no universal consensus for its implementation.

\footnotetext{
${ }^{1}$ Banco de Sangre del Instituto Nacional de Pediatría, Secretaría de Salud, México.

${ }^{2}$ Centro Nacional de Equidad de Género y Salud Reproductiva, Secretaría de Salud, México.

${ }^{3}$ Unidad de Genética de la Nutrición, Instituto de Investigaciones Biomédicas, UNAM, México.

${ }^{4}$ Laboratorio de Errores Innatos del Metabolismo y Tamiz, Instituto Nacional de Pediatría, Secretaría de Salud, México. ${ }^{5}$ TamizMas Screening Center de Químicos Maldonado, Mérida, Yucatán.
}

Correspondencia

Erika Paola García Flores

erika.garcia@salud.gob.mxT

Este artículo debe citarse como Maldonado Silva K, Hinojosa Trejo MA, Ibarra González I, Vela Amieva M, Herrera Pérez LA, Caamal Parra G, Sulu Huicab JE, García Flores EP. Valores de glucosa-6-fosfato deshidrogenasa y su repercusión en el número de sospechas de tamiz neonatal.Acta Pediatr Mex. 2018;SI(39):47S-57S. 
OBJECTIVE: To describe the enzymatic activity of G6PD in newborn (NB) bloodspot filter paper specimens, to analyze the prevalence and estimate the number of locations that must be performed in the NBS program.

MATERIAL AND METHODS: Retrospective study of the results of the NBS program of the Ministry of Health of Mexico. The enzymatic activity of G6PD was analyzed according on the sample transit time (time elapsed from obtaining the blood sample from the NB heel, until his arrival at the laboratory).

RESULTS: A total of 1,076,918 RN were screened, 342,272 had adequate transit time ( $\leq 6$ days). Enzymatic activity decreases significantly after that Time. The national birth prevalence was $4.26 \%$, with a maximum in Veracruz, Nuevo León and Tabasco (21, 20 and $15 \%$, respectively).

CONCLUSION: In the Ministry of Health of Mexico, $4.26 \%$ of the NB are dG6PD this is, one case for every 23 screened, which implies a high number of subjects to locate and evaluate in the institutions that carry out the clinical follow-up.

KEYWORDS: Glucose 6-phosphate, glucose-6-phosphate deficiency, newborn screening, prevalence.

\section{INTRODUCCIÓN}

La glucosa-6-fosfato deshidrogenasa (G6PD, EC 1.1.1.49) es la primera enzima de la vía de las pentosas fosfato, una vía metabólica altamente conservada, responsable de la producción de una variedad de moléculas decisivas, incluidos precursores de nucleótidos y nicotinamida-adenina-dinucleótido-fosfato (NADPH). En la primera reacción de la fase oxidativa de la vía de las pentosas-fosfato, la G6PD cataliza la conversión de glucosa-6-fosfato a 6-fosfogluconolactona con la producción concomitante de una molécula de NADPH. Cuando el 6-fosfogluconato se convierte en ribosa-5-fosfato por la 6-fosfogluconato deshidrogenasa se produce una segunda molécula de NADPH. ${ }^{1,2}$ La G6PD tiene un papel fundamental en la defensa antioxidante del eritrocito, puesto que, si las concentraciones de NADPH no pueden conservarse, el glutatión reducido disminuye y ocurre daño oxidativo que desemboca finalmente en hemólisis. ${ }^{3}$

La deficiencia de G6PD (dG6PD) se considera la enzimopatía hereditaria más frecuente en el mundo. ${ }^{4,5}$ Es un rasgo monogénico con un patrón de herencia recesivo ligado al cromosoma $X$, en el que los varones hemicigotos para alelos deficientes del gen responsable tienen riesgo de anemia hemolítica por dG6PD. Mientras que las mujeres pueden ser normales o deficientes por un genotipo hemicigotos o heterocigoto compuesto para alelos hipomorfos que les condiciona riesgo de anemia hemolítica por la deficiencia enzimática. En general, las mujeres heterocigotas (portadoras) se asumen sin riesgo de padecer anemia hemolítica por dG6PD. ${ }^{6}$ El gen de la G6PD (MIM *305900) consta de 13 exones y 12 intrones organizados en 16.2 kilobases en $\mathrm{Xq} 28$.

El cuadro clínico de la dG6PD es resultado del estrés oxidativo generado por factores desencadenantes. Debido al bajo nivel de glutatión en grupos sulfidrilos, que no pueden mantenerse en su forma reducida, se producen uniones intra e intermoleculares entre estos grupos, con la formación de agregados de proteínas del citoesqueleto de la membrana del eritrocito, estructuras (cuerpos de Heinz) que disminuyen la deformabilidad y alteran la superficie celular y provocan que los macrófagos las reconozcan como extrañas dando lugar a la hemólisis. Además, se produce hemólisis oxidativa debido a la incapacidad del eritrocito para eliminar de manera eficiente los radicales libres de hidrógeno y oxígeno. ${ }^{7,8}$ 
La OMS clasifica la dG6PD en cinco categorías según la severidad clínica de la deficiencia enzimática. ${ }^{9}$ (Cuadro 1) Los individuos con deficiencia de dG6PD suelen permanecer asintomáticos toda su vida, excepto los casos esporádicos con deficiencia clase I (actividad enzimática menor de $10 \%){ }^{8}$

Las manifestaciones clínicas incluyen un grupo de condiciones hematológicas, como: anemia hemolítica aguda, la ictericia neonatal y anemia hemolítica crónica no esferocítica crónica (AHCNE). ${ }^{10}$ En el mundo las más frecuentes son la ictericia neonatal y la anemia hemolítica aguda, que generalmente se desencadena por un agente exógeno. (Cuadro 2) Algunas variantes de G6PD causan hemólisis crónica, que da lugar a AHCNE.? En Latinoamérica las principales manifestaciones clínicas reportadas de una revisión sistemática fueron las relacionadas con anemia hemolítica aguda, sobre todo hemólisis inducida por fármacos; la mayoría son casos de crisis hemolíticas en pacientes con malaria por Plasmodium vivax durante el tratamiento con primaquina. El favismo, la hemólisis inducida por infección, la ictericia neonatal y la AHCNE parecen jugar un papel menor en los problemas de salud pública de Latinoamérica y en la

Cuadro 1. Clasificación de la Organización Mundial de la Salud (OMS) de las variantes de la deficiencia de dG6PD

\begin{tabular}{l|c|c|}
\hline Clase & $\begin{array}{c}\text { Actividad } \\
\text { enzimática }\end{array}$ & \multicolumn{1}{c|}{ Características clínicas } \\
\hline I & $<10 \%$ & $\begin{array}{l}\text { Los pacientes pueden tener hemólisis } \\
\text { espontánea, sin estresor oxidativo. } \\
\text { Puede asociarse con anemia hemo- } \\
\text { lítica crónica no esferocítica grave. }\end{array}$ \\
\hline II & $<10 \%$ & $\begin{array}{l}\text { Puede o no cursar con episodios } \\
\text { severos de anemia hemolítica no } \\
\text { esferocítica. }\end{array}$ \\
\hline III & $10-60 \%$ & $\begin{array}{l}\text { Puede o no cursar con episodios } \\
\text { moderados o leves de anemia he- } \\
\text { molítica, usualmente asintomáticos. }\end{array}$ \\
\hline IV & $60-100 \%$ & $\begin{array}{l}\text { Usualmente asintomáticos. } \\
\text { Únicamente un caso reportado en } \\
\text { el mundo. }\end{array}$ \\
\hline V & $>100 \%$
\end{tabular}

mayoría de los casos la deficiencia no afecta la calidad, actividad o esperanza de vida. ${ }^{7}$

Cuadro 2. Factores desencadenantes de hemólisis

\begin{tabular}{|c|c|}
\hline Grupo & Tiро \\
\hline Alimentos & $\begin{array}{l}\text { Habas (Vicia faba), en todas sus } \\
\text { formas. }\end{array}$ \\
\hline \multirow[t]{6}{*}{ Medicamentos } & $\begin{array}{l}\text { Antimaláricos con riesgo definitivo: } \\
\text { - Primaquina } \\
\text { - Combinaciones que contienen } \\
\text { dapsona y pamaquinaxt }\end{array}$ \\
\hline & $\begin{array}{l}\text { Antimaláricos con riesgo posible: } \\
\text { - Cloroquina } \\
\text { - Quinidina } \\
\text { - Quinina }\end{array}$ \\
\hline & $\begin{array}{l}\text { Otros medicamentos con riesgo } \\
\text { definitivo: }\end{array}$ \\
\hline & $\begin{array}{l}\text { - Azul de metileno (cloruro de } \\
\text { metiltionina) } \\
\text { - Ciprofloxacina } \\
\text { - Moxifloxacina } \\
\text { - Ácido nalidíxico } \\
\text { - Niridazol } \\
\text { - Nitrofurantoína } \\
\text { - Norfloxacina } \\
\text { - Ofloxacina } \\
\text { - Sulfametoxazol/cotrimoxazol } \\
\text { - Rasburicasa }\end{array}$ \\
\hline & $\begin{array}{l}\text { Otros medicamentos con riesgo } \\
\text { posible: }\end{array}$ \\
\hline & $\begin{array}{l}\text { - Ácido acetilsalicílico (aspirina) } \\
\text { - Sulfadiazina } \\
\text { - Sulfasalazina } \\
\text { - Sulfonilureas } \\
\text { - Cloranfenicol } \\
\text { - Ácido dimercaptosuccínico } \\
\text { - Glibenclamida } \\
\text { - Mepacrina } \\
\text { - Análogos de la vitamina K }\end{array}$ \\
\hline $\begin{array}{l}\text { Productos } \\
\text { químicos }\end{array}$ & $\begin{array}{l}\text { Naftaleno (bolitas antipolillas de } \\
\text { naftalina y alcanfor) }\end{array}$ \\
\hline \multirow[t]{4}{*}{ Infecciones } & Virales \\
\hline & $\begin{array}{l}\text { - Hepatitis A y B } \\
\text { - Citomegalovirus }\end{array}$ \\
\hline & Bacterianas \\
\hline & $\begin{array}{l}\text { - Neumonías } \\
\text { - Fiebre tifoidea } \\
\text { - Rickettsiosis (Tifus). }\end{array}$ \\
\hline Físicos & Ejercicio vigoroso. \\
\hline
\end{tabular}


Ictericia neonatal: La dG6PD es uno de los principales factores de riesgo de ictericia neonatal severa. ${ }^{12}$ Suele aparecer en las primeras 24 horas de vida, antes que la ictericia fisiológica, pero más tarde en comparación con la ictericia que se observa en la aloinmunización a grupo sanguíneo. Los recién nacidos con dG6PD pueden tener, además de la ictericia neonatal, problemas para la alimentación, letargo, dificultad para respirar o convulsiones. Cuando la dG6PD se asocia con prematurez, infección, factores ambientales (bolitas de naftalina-alcanfor que se usa en la ropa para evitar las polillas) o coexistencia de galactosemia, la ictericia puede ser muy grave. ${ }^{7}$ ${ }^{13}$ La dG6PD es la principal causa identificada de hiperbilirrubinemia crítica (definida como una bilirrubina sérica total superior a $30 \mathrm{mg} / \mathrm{dL}$ ), sobre todo en regiones con elevada prevalencia. ${ }^{14,15} \mathrm{Si}$ no se trata adecuadamente, la ictericia neonatal asociada con la deficiencia de G6PD puede producir kernícterus o encefalopatía bilirrubínica, con daño neurológico permanente. ${ }^{16,17}$

Anemia hemolítica aguda: es de carácter episódico agudo y ocurre cuando el individuo se expone al estrés oxidativo causado por desencadenantes (Cuadro 2). La hemólisis se manifiesta 24-72 horas después de la exposición y, en casos de hemólisis grave, los pacientes tienen síntomas y signos de anemia moderada a grave, fiebre, malestar general, irritabilidad, debilidad, ictericia y coluria. El inicio puede ser extremadamente abrupto, especialmente con el favismo en los niños. ${ }^{18}$ La hemólisis aguda suele desaparecer espontáneamente y se resuelve entre 8 a 14 días, debido a la producción compensatoria de eritrocitos jóvenes. La insuficiencia renal aguda por necrosis tubular y la coagulación intravascular diseminadas son complicaciones raras de la anemia hemolítica aguda. ${ }^{19}$

\section{Anemia hemolítica crónica no esferocítica:} se manifiesta en los individuos con variantes de clase I (por ejemplo, Brighton, Harilaou y
Serres), que implican deficiencia enzimática grave. La anemia hemolítica es de grado variable y la hemólisis es sólo parcialmente intravascular. Se puede acompañar de cálculos biliares y esplenomegalia. Sin embargo, existe una gran variabilidad en las manifestaciones asociadas con este tipo de anemia crónica. . $^{18,20}$

En algunos países, particularmente en Asia y el Mediterráneo y en los de elevado número de casos de malaria, se ha considerado la realización del tamiz neonatal para la dG6PD; ${ }^{21,22}$ sin embargo, la mayor parte de los países industrializados no llevan a cabo este tamiz. ${ }^{12,23}$ En México existen algunos estudios para la detección de la deficiencia de la enzima en distintas instituciones de salud pública y la epidemiología de la dG6PD la han analizado diversos autores en grupos reducidos de individuos y se han efectuado en regiones geográficas limitadas. ${ }^{24-27}$

El objetivo de este estudio fue: describir los valores de la actividad de la enzima G6PD cuantificada en papel filtro en recién nacidos mexicanos y analizar la repercusión en el número de localizaciones a efectuar en el marco de un programa de tamiz neonatal nacional.

\section{MATERIALES Y MÉTODOS}

Estudio retrospectivo de los resultados del programa de tamiz neonatal del CNEGSR de la Secretaría de Salud de México efectuado del 20 de mayo de 2017 al 31 de mayo de 2018. Las muestras de sangre se obtuvieron mediante punción del talón y se recolectaron en papel filtro especial (tarjeta de Guthrie). Después de la recolección los especímenes se enviaron por mensajería al laboratorio procesador adjudicado por el CNEGSR.

Se analizaron los valores de la actividad de la G6PD, dependiendo del tiempo de tránsito (tiempo trascurrido desde la obtención de la muestra de sangre del talón del recién nacido hasta su 
llegada al laboratorio procesador), categorizándolas en dos grupos: 1) Tiempo de tránsito igual o menor a 6 días y, grupo 2) Tiempo de tránsito mayor de 6 días. También se analizaron los resultados tomando en cuenta la nueva clasificación de Luzzato y sus colaboradores, según la actividad enzimática residual. ${ }^{8}$

Procesamiento bioquímico. Todas las muestras se procesaron en las 24 horas siguientes a su recepción en el laboratorio. Se utilizaron equipos automatizados Panthera-puncher- $9^{\circledR}$ de PerkinElmer $^{\circledR}$ para perforar las muestras y se obtuvieron círculos de sangre seca estandarizados de 3.2 mm para el análisis bioquímico. La determinación cuantitativa in vitro en sangre en papel filtro se efectuó mediante método de microplaca fluorescente el estuche comercial GSP ${ }^{\circledR} \mathrm{Neo}-$ natal G6PD y equipos automatizados Genetic Screening Processor $^{\circledR}\left(\mathrm{GSP}^{\circledR}\right)$ de PerkinElmer ${ }^{\circledR}$. Las unidades en las que se expresaron los resultados fueron $\mathrm{U} / \mathrm{dL}$, que asumen $3.42 \mu \mathrm{L}$ de sangre en cada confeti de $3.2 \mathrm{~mm}$ y que minimizan la variabilidad de la hemoglobina neonatal.

Consideraciones éticas. Las muestras solo se tomaron con consentimiento verbal debido a que el tamiz neonatal es una acción obligatoria para todas las unidades médicas que atiendan personas recién nacidas, de conformidad con la normatividad vigente. ${ }^{28-31}$

Análisis estadístico. Para los datos numéricos se utilizaron medidas de tendencia central: promedio, mediana y percentiles, intervalos y porcentajes. Se utilizó el programa de acceso libre Comprehensive R Archive Network (https:// cran.r-project.org).

\section{RESULTADOS}

De 1,076,918 recién nacidos tamizados, 343,272 cumplieron con el tiempo de tránsito adecuado $(\leq 6$ días). En la Figura 1 se muestra el efecto que el tiempo de tránsito tiene en la actividad de G6PD en sangre neonatal; la actividad disminuye significativamente si las muestras se procesan después de 6 días de haberse obtenido. La mediana del grupo con tiempo de tránsito adecuado fue de $53.3 \mathrm{U} / \mathrm{dL}$ y la del grupo con tiempo de tránsito prolongado fue de $41.93 \mathrm{U} / \mathrm{dL}$.

La distribución de la actividad de G6PD analizada en las 343,272 muestras con tiempo adecuado de tránsito y categorizada por sexo se presenta en la Figura 2; se observa que es distinta en las mujeres con percentil $0.5 \%$ que corresponde a una actividad residual de 19.38 $\mathrm{U} / \mathrm{dL}$, mientras que en los hombres el mismo percentil corresponde a una actividad de 9.68 $\mathrm{U} / \mathrm{dL}$; de igual manera, la mediana entre ambos grupos $(53.48 \mathrm{U} / \mathrm{dL}$ en mujeres y $52.83 \mathrm{U} / \mathrm{dL}$ en hombres) es significativamente diferente $(\mathrm{p}<$ 0.0001).

En el Cuadro 3 se reporta la prevalencia de la dG6PD por entidad federativa y nacional, considerando diversos grados de actividad enzimática residual y el tiempo de tránsito ideal ( $\leq 6$ días). La prevalencia nacional fue de $4.26 \%$, esto es:

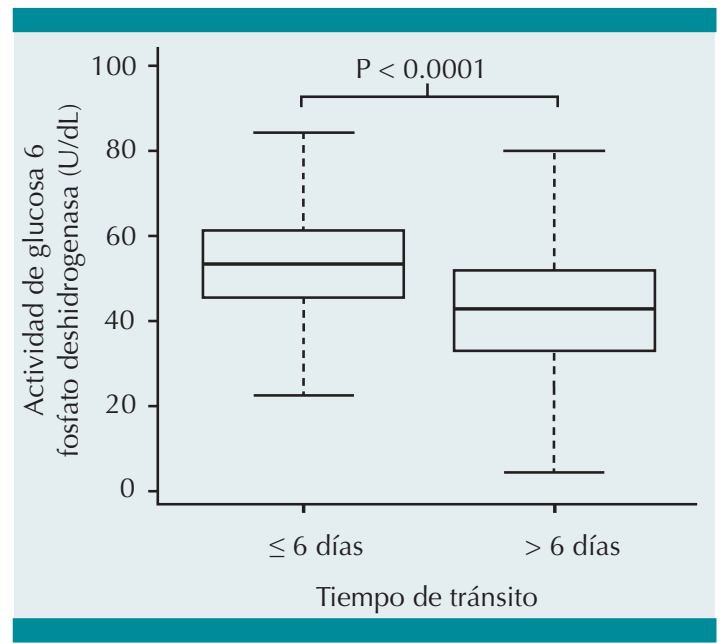

Figura 1. Efecto del tiempo de tránsito de las muestras en la actividad de la enzima G6PD ( $n=1,076,918$ muestras). 


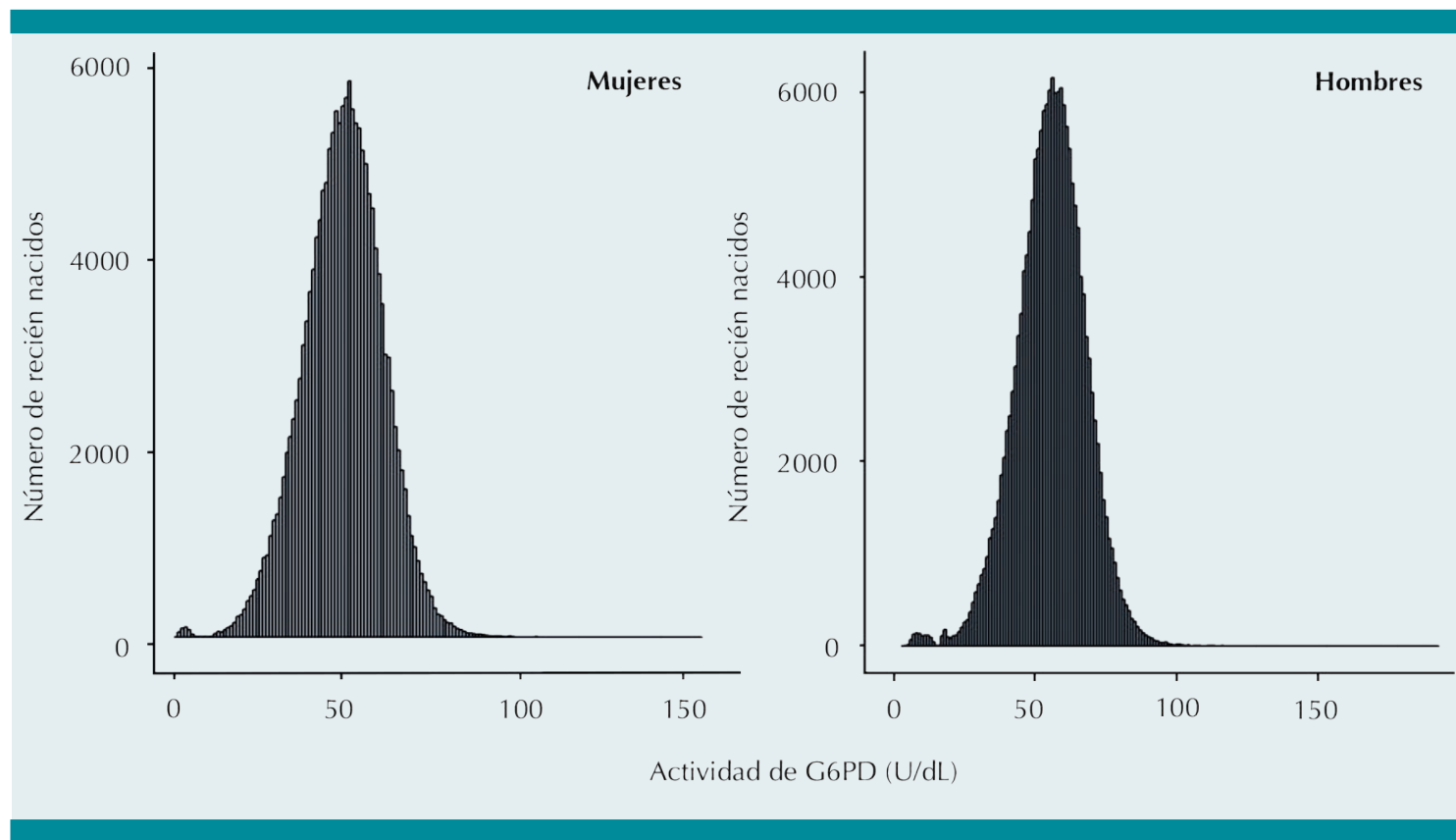

Figura 2. Distribución de los valores de actividad de la enzima G6PD en una submuestra de niñas $(n=137,585)$ y niños $(n=142,433)$ tamizados.

un caso por cada 23 recién nacidos tamizados. Los estados de mayor prevalencia fueron Veracruz, Nuevo León y Tabasco con 21, 20 y $15 \%$, respectivamente. En todos los estados se encontraron casos (Figura 3).

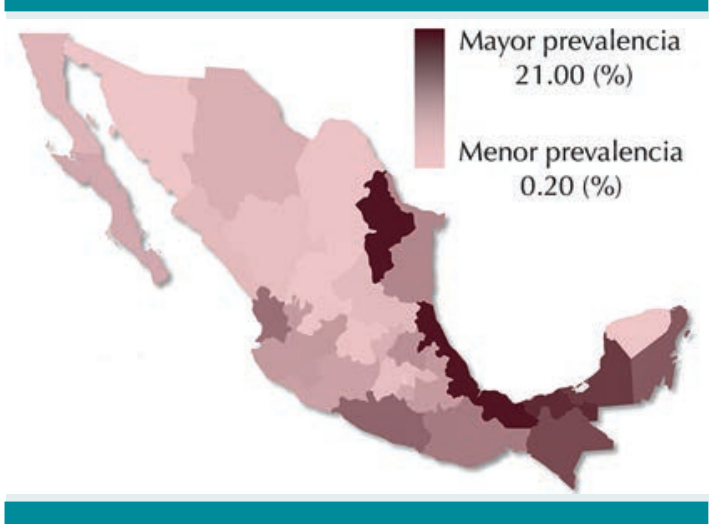

Figura 3. Distribución de la prevalencia (\%) de G6PD en México.
El 95\% de los individuos deficientes fueron niños, $4.4 \%$ niñas y en $0.5 \%$ de los casos el dato de sexo no se consignó en la ficha demográfica.

\section{DISCUSIÓN}

Se reportaron las distribuciones y características de los valores de la actividad de la enzima G6PD en sangre en una población de más de un millón de recién nacidos mexicanos tamizados mediante ensayo inmunofluorométrico.

El primer hallazgo importante de este trabajo fue que el número de muestras que cumplen con el tiempo de tránsito adecuado ( $\leq 6$ días) fue extremadamente bajo (343,272 muestras, 31.88\%) y se demostró, al igual que otros autores, que la actividad de la G6PD desciende significativamente después de ese tiempo. ${ }^{32}$ Interpretar los resultados sin tomar en cuenta este factor 


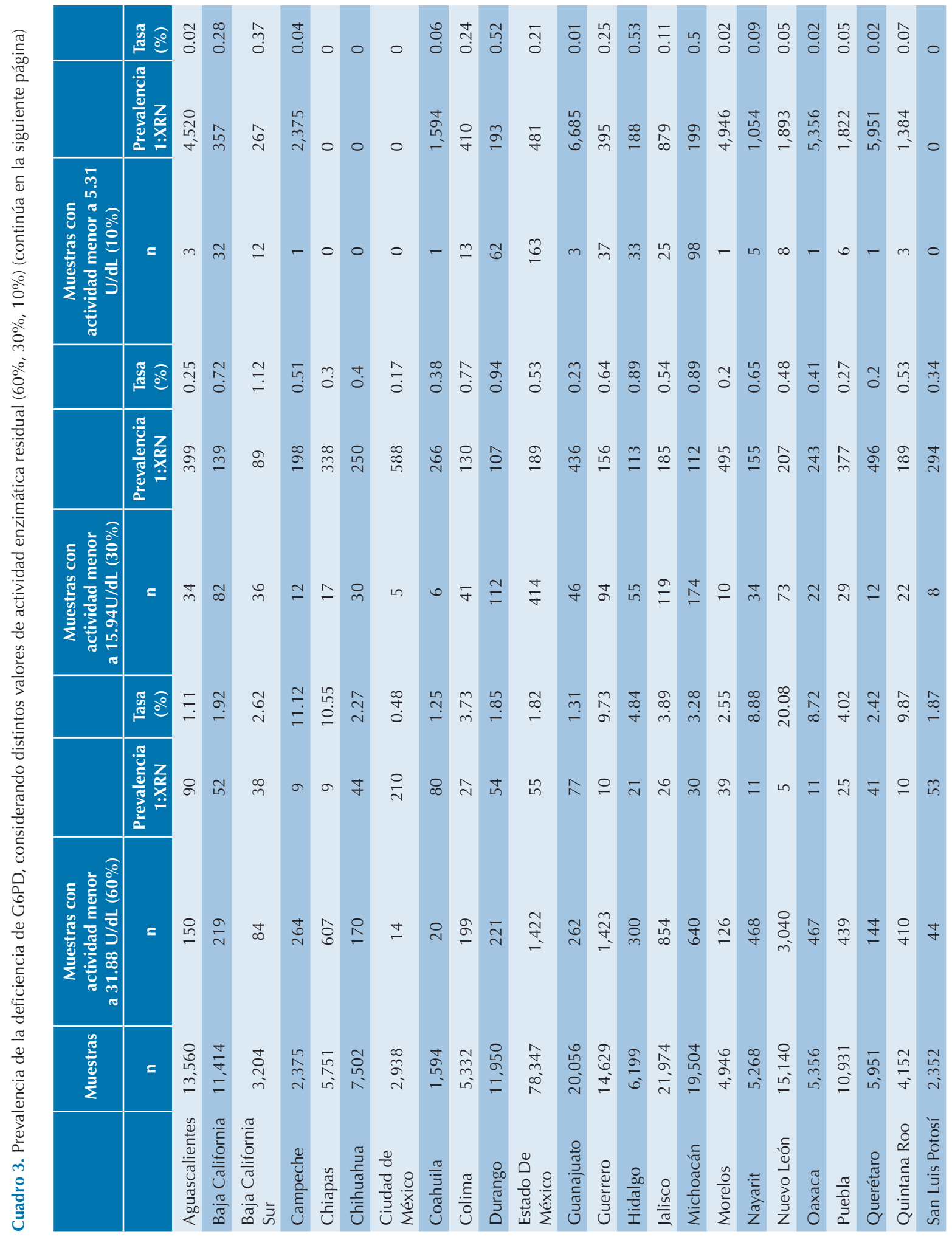




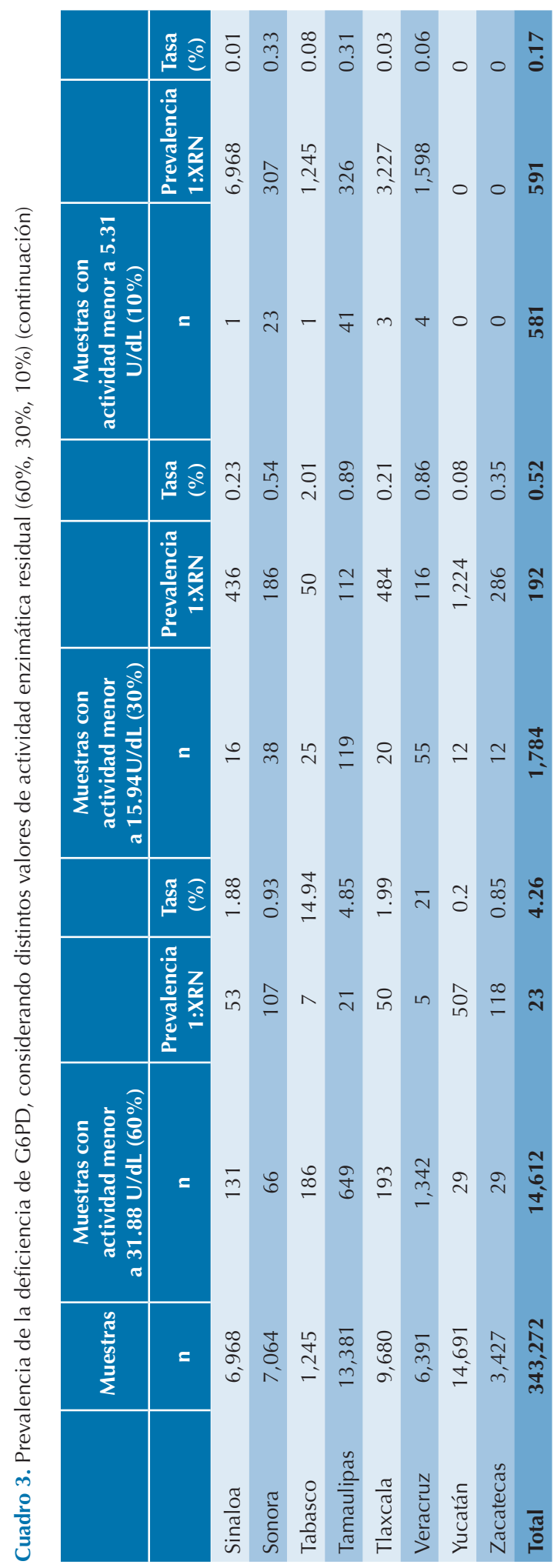

es un error que sobreestima de manera ficticia la prevalencia de la enfermedad, con todos los problemas que esto implica: saturación de los servicios de salud, estudios innecesarios para los recién nacidos y, sobre todo, la angustia generada en las familias. ${ }^{23}$ La incorporación de esta enfermedad a los paneles de tamiz implica retos muy importantes a considerar, entre los que destaca la repercusión en el número de localizaciones que se tienen que realizar porque es un padecimiento genético muy frecuente que, en algunas regiones del país, afecta incluso a más de 20\% de la población (Cuadro 3, Figura 3). En el mundo se han implementado algunos programas de tamiz neonatal para la dG6PD pero dichos países suelen tener la característica de ser étnicamente homogéneos y endémicos para malaria. ${ }^{21}$ En países multiétnicos, como Estados Unidos o Canadá, existen debates y argumentos, incluido el costo-efectividad, para realizar el tamiz de dG6PD ${ }^{17,33}$ así como la ausencia de datos sólidos publicados que determinen la relevancia clínica de la enfermedad. ${ }^{23}$

Algunos autores recomiendan que el tamiz para G6PD se lleve a cabo de manera sistemática solo en recién nacidos con ictericia y en pacientes con hemólisis no inmunológica; es decir, recién nacidos de alto riesgo y adultos que van a recibir terapia con primaquina. ${ }^{7,8,34}$

Además, hay que considerar que puesto que es una enfermedad con herencia ligada al cromosoma X, la actividad de la G6PD es diferente en los hombres que en las mujeres (Figura 2). Esta característica es relevante puesto que como señalan otros autores tiene influencia en el valor de corte del tamiz, mismo que debe categorizarse por sexo. ${ }^{22,35}$

En México se han efectuado contribuciones significativas al conocimiento de la deficiencia de G6PD, en particular en sus aspectos funcionales, bioquímicos y epidemiológicos. ${ }^{2,36,37}$ La 
prevalencia encontrada por nosotros $(4.26 \%)$ coincide con lo publicado para México $(0.39 \%$ $4.09 \%)^{38}$ y es semejante a la reportada en China $(4.2-4.5 \%) .{ }^{39,40}$

De 343,272 recién nacidos, 14,612 muestras tuvieron una actividad enzimática menor de $60 \%(31.88 \mathrm{U} / \mathrm{Ld})$ y que, de acuerdo con la clasificación de la OMS, abarcarían las clases I, II y III. Luzzato y Capellini propusieron una reclasificación de la deficiencia de dG6PD considerando la actividad enzimática residual; sin embargo, ninguna de estas clasificaciones ha demostrado ser útil para los casos que se detectan mediante el tamiz neonatal. 7,8 Todos estos individuos requieren seguimiento clínico para conocer la historia natural de la enfermedad y poder tomar mejores decisiones de salud pública. ${ }^{12,23}$

Los resultados de este trabajo demuestran la importancia de establecer un valor de corte diferenciado por sexo para el tamiz neonatal de la dG6PD, estiman el número de localizaciones dependiendo de la severidad de la deficiencia de la actividad enzimática y permiten dimensionar la trascendencia de practicar esta prueba en el contexto del programa nacional de tamiz.

\section{CONCLUSION}

En México, 4.26\% de los recién nacidos tienen dG6PD esto es, un caso por cada 23 tamizados, lo que implica un elevado número de sujetos para localizar y evaluar en los programas de tamiz neonatal y en las instituciones que llevan a cabo el seguimiento clínico.

\section{REFERENCIAS}

1. Cunningham AD, Hwang $S$, Mochly-Rosen D. Glucose-6-Phosphate Dehydrogenase Deficiency and the Need for a Novel Treatment to Prevent Kernicterus. Clin Perinatol. 2016;43(2):341-54.

2. Gómez-Manzo S, et al. Functional and Biochemical Characterization of Three Recombinant Human Glucose-6-Phos- phate Dehydrogenase Mutants: Zacatecas, Vanua-Lava and Viangchan. Int J Mol Sci. 2016;17 pii: E787. doi: 10.3390/ ijms17050787.

3. Mason PJ, Bautista JM, Gilsanz F. G6PD deficiency: the genotype-phenotype association. Blood Reviews. 2007;21(5):267-283.

4. Hirono A, Kanno H, Miwa S, Beutler E. Pyruvate kinase deficiency and other enzymopathies of the erythrocyte. In Scriver CR, Beaudet AL, Sly WS et al, eds. The Metabolic \& Molecular Bases of Inherited Disease. New York: McGraw-Hill, 2001;4637-64.

5. Nkhoma ET, Poole C, Vannappagari V, Hall SA, Beutler E. The global prevalence of glucose-6-phosphate dehydrogenase deficiency: a systematic review and meta-analysis. Blood Cells Mol Dis. 2009;42(3):267-78.

6. Luzzatto L, Mehta A, Vulliamy T. Glucose-6-phosphate dehydrogenase deficiency. In: Scriver CR, et al. The Metabolic and Molecular Basis of Inherited Disease. New York: McGraw-Hill 2013: 4517-53.

7. Cappellini M, Fiorelli G. Glucose-6-phosphate dehydrogenase deficiency. The Lancet. 2008; 371(9606):64-74.

8. Luzzatto L, et al. Glucose-6-Phosphate Dehydrogenase Deficiency. Hematol Oncol Clin North Am. 2016;30(2):373-93. doi: 10.1016/j.hoc.2015.11.006

9. WHO working group. Glucose-6-phosphate dehydrogenase deficiency. Bull World Health Organ 1989; 67(6): 601-11

10. Ong KIC, et al. Systematic review of the clinical manifestations of glucose-6-phosphate dehydrogenase deficiency in the Greater Mekong Subregion: implications for malaria elimination and beyond. BMJ Glob Health. 2017 19;2(3):e000415. doi: 10.1136/bmjgh-2017-000415

11. Monteiro WM, et al. G6PD deficiency in Latin America: systematic review on prevalence and variants. Mem Inst Oswaldo Cruz. 2014;109(5):553-68.

12. American Academy of Pediatrics. Subcommittee of hyperbilirubinemia: management of hyperbilirubinemia in the newborn infant 35 or more weeks of gestation. Pediatrics. 2004;114(1):297-316.

13. Santucci K, Shah B. Association of naphthalene with acute hemolytic anemia. Acad Emerg Med. 2000;7(1):42-7.

14. Kuzniewicz MW, et al. Incidence, etiology, and outcomes of hazardous hyperbilirubinemia in newborns. Pediatrics. 2014;134(3):504-9. doi: 10.1542/peds.2014-0987.

15. Al-Omran A, Al-Abdi S, Al-Salam Z. Readmission for neonatal hyperbilirubinemia in an area with a high prevalence of glucose-6-phosphate dehydrogenase deficiency: A hospital-based retrospective study. J Neonatal Perinatal Med. 2017; 10(2):181-9.

16. Weng $\mathrm{YH}$, et al. Clinical characteristics of G6PD deficiency in infants with marked hyperbilirubinemia. J Pediatr Hematol Oncol. 2010;32(1):11-4. doi: 10.1097/MPH.0b013e3181c09aec.

17. Kaplan $\mathbf{M}$, et al. Neonatal screening for glucose-6-phosphate dehydrogenase deficiency: biochemical versus ge- 
netic technologies. Semin Perinatol. 2011 Jun;35(3):155-61. doi: 10.1053/j.semperi.2011.02.010.

18. Beutler E. Glucose-6-phosphate dehydrogenase deficiency: a historical perspective. Blood. 2008;111(1):16-24.

19. Arese P, De Flora A. Pathophysiology of hemolysis in glucose 6-phosphate dehydrogenase deficiency. Semin Hematol 1990;27(1):1-40.

20. Luzzatto L, Seneca E. G6PD deficiency: a classic example of pharmacogenetics with on-going clinical implications. $\mathrm{Br} J$ Haematol 2014;164(4):469-80.

21. Goyal M, Garg A, Goyal MB, Kumar S, Ramji S, Kapoor S. Newborn screening for G6PD deficiency: A 2-year data from North India. Indian J Public Health. 2015;59(2):145-8.

22. Fu C, et al. Newborn screening of glucose-6-phosphate dehydrogenase deficiency in Guangxi, China: determination of optimal cutoff value to identify heterozygous female neonates. Sci Rep. 2018;8(1):833. doi: 10.1038/s41598017-17667-6.

23. American College of Medical Genetics (ACMG)/Health Resources and Service Administration (HRSA). 2005. Newborn screening: Toward a uniform screening panel and system. http://mchb.hrsa.gov/screening/.

24. Vaca G, Arámbula E, Esparza A. Molecular heterogeneity of glucose-6-phosphate dehydrogenase deficiency in Mexico: overall results of a 7-year project. Blood Cells Mol Dis. 2002;28(3):436-44.

25. Vaca G y Arámbula ME. DNA sequencing analysis of several G6PD variants previously defined by PCR restriction enzyme analysis. Genet Mol Biol. 2006; 29(1):31-5

26. Zamorano-Jiménez CA y col. Identificación molecular de la glucosa-6-fosfato deshidrogenasa (G6PD) detectada en el tamiz neonatal. Gac Med Mex. 2015; 151(1):34-41.

27. Trigo-Madrid M, Díaz-Gallardo J, Mar-Aldana R, Ruiz-Ochoa D, Moreno-Graciano C, Martínez-Cruz P, Herrera-Pérez LA, et al. Resultados del Programa de Tamiz Neonatal Ampliado y epidemiología perinatal en los servicios de sanidad de la Secretaría de Marina Armada de México. Acta Pediatr Mex 2014; 35(6): 448-58.

28. Ley General de Salud (2007). Disponible: http://www.salud. gob.mx/unidades/cdi/legis/lgs/index-indice.htm.

29. Norma Técnica número 321 para la prevención del retraso mental producido por hipotiroidismo congénito. Diario Oficial de la Federación, 22/09/1988, México.
30. Norma Oficial Mexicana NOM-034-SSA2-2013, Para la prevención y control de los defectos al nacimiento. Diario Oficial de la Federación, 24/06/2014, México.

31. Norma Oficial Mexicana NOM-007-SSA2-2016, Para la atención de la mujer durante el embarazo, parto y puerperio, y de la persona recién nacida. Diario Oficial de la Federación, 01/04/2016, México.

32. Kuwahata $M$, et al. Population screening for glucose-6-phosphate dehydrogenase deficiencies in Isabel Province, Solomon Islands, using a modified enzyme assay on filter paper dried bloodspots. Malar J. 2010;9:223. doi: 10.1186/1475-2875-9-223.

33. Sirdah MM, et al. National G6PD neonatal screening program in Gaza Strip of Palestine: rationale, challenges and recommendations. Clin Genet. 2016;90(3):191-8. doi: 10.1111/cge.12786.

34. Vaca 1982 Vaca G, Ibarra B, Romero F, Olivares N, Cantú JM, Beutler E. G-6-PD Guadalajara. A new mutant associated with chronic nonspherocytic hemolytic anemia. Hum Genet. 1982;61(2):175-6.

35. Miao JK, Chen QX, Bao LM, Huang Y, Zhang J, Wan KX, et al. Determination of optimal cutoff value to accurately identify glucose-6-phosphate dehydrogenase-deficient heterozygous female neonates. Clin Chim Acta. 2013;17(12): 131-5.

36. Lisker R, Loria A, Cordova Ms. Studies on several genetic hematological traits of the Mexican population. Hemoglobin s, glucose-6-phosphate dehydrogenase deficiency, and other characteristics in a malarial region. Am J Hum Genet. 1965;17(2):179-87.

37. Gómez-Manzo S, et al. Glucose-6-Phosphate Dehydrogenase: Update and Analysis of New Mutations around the World. Int J Mol Sci. 2016;17: pii E2069.

38. Medina MD, Vaca G, Lopez-Guido B, Westwood B, Beutler E. Molecular Genetics of Glucose-6-Phosphate Dehydrogenase Deficiency in Mexico. Blood Cells, Molecules, and Diseases.1997;23(5): 88-94.

39. Jiang J, et al. Screening and prevention of neonatal glucose 6-phosphate dehydrogenase deficiency in Guangzhou, China. Genet Mol Res. 2014;13(2):4272-9. doi: 10.4238/2014.June.9.13.

40. Lam ST, Cheng ML. Neonatal screening in Hong Kong and Macau. Southeast Asian J Trop Med Public Health. 2003;34 Suppl 3:73-5. 